CERN-2000-029

14 February 2000

\title{
Measurement of the electric quadrupole moments of ${ }^{26-29} \mathrm{Na}$
}

\author{
M. Keim $₫$, U. Georg ${ }^{\dagger}$, A. Klein ${ }^{\dagger}$, R. Neugart ${ }^{\dagger}$, M. Neuroth ${ }^{\dagger}$, S. Wilbert ${ }^{\dagger}$, P. Lievens ${ }^{\ddagger}$, \\ L. Vermeeren*, B.A. Brown ${ }^{+}$and the ISOLDE Collaboration \\ † Institut für Physik, Universität Mainz, D-55099 Mainz, Germany \\ $\ddagger$ Laboratorium voor Vaste-Stoffysica en Magnetisme, \\ K. U. Leuven, B-3001 Leuven, Belgium \\ * Instituut voor Kern- en Stralingsfysica, K. U. Leuven, B-3001 Leuven, Belgium \\ + Department of Physics and Astronomy and NSCL, \\ Michigan State University, East Lansing, MI 48824, USA \\ ^ EP Division, CERN, CH-1211 Geneva 23, Switzerland
}

IS 304

\begin{abstract}
The nuclear electric quadrupole moments of the isotopes ${ }^{26} \mathrm{Na},{ }^{27} \mathrm{Na},{ }^{28} \mathrm{Na}$ and ${ }^{29} \mathrm{Na}$ were measured by $\beta$-NMR spectroscopy in single crystals of $\mathrm{LiNbO}_{3}$ and $\mathrm{NaNO}_{3}$. High degrees of nuclear polarization were produced by optical pumping of the sodium atoms in a fast beam with a collinear laser beam. The polarized nuclei were implanted into the crystals and NMR signals were observed in the $\beta$-decay asymmetries. Preparatory measurements also yielded improved values for the magnetic moments of ${ }^{27-31} \mathrm{Na}$ and confirmed the spin $I=3 / 2$ for ${ }^{31} \mathrm{Na}$. The results are discussed in comparison with large-basis shell-model calculations.
\end{abstract}

PACS: $21.10 . \mathrm{Ky} ; 27.30 .+\mathrm{t} ; 76.60 .-\mathrm{k}$ 


\section{Introduction}

Measurements of the small quadrupole moments of light nuclei require appropriate experimental techniques. Most quadrupole moments of nuclear ground states were obtained from measurements of the hyperfine structure of atomic energy levels. However, the quadrupole moments and even more the atomic hyperfine fields decrease with the atomic charge number $Z$. As a result, the quadrupole interaction contributes extremely little to the hyperfine structure of low- $Z$ atomic systems.

For sodium the most easily accessible state for quadrupole interaction studies is $3 \mathrm{p}^{2} \mathrm{P}_{3 / 2}$, reached from the $3 \mathrm{~s}^{2} \mathrm{~S}_{1 / 2}$ ground state by excitation in the D2 line. Here the resolution is limited by the natural line width of $10 \mathrm{MHz}$ which exceeds the quadrupole interaction by about one order of magnitude. Former experiments on the isotopes ${ }^{21} \mathrm{Na}$ and ${ }^{25-29} \mathrm{Na}$ made an attempt to resolve this small effect by employing laser-rf double resonance spectroscopy [1]. However, the experimental errors of about $50 \mathrm{mb}$ were of the same order as the absolute values of the measured quadrupole moments.

The experiments on ${ }^{26-29} \mathrm{Na}$ presented here were performed by $\beta$-ray detected nuclear magnetic resonance spectroscopy ( $\beta$-NMR, see ref. [2]) on nuclei polarized by in-beam optical pumping [3]. This technique has already been used to measure the quadrupole moments of ${ }^{9} \mathrm{Li}$ and ${ }^{11} \mathrm{Li}[4,5]$. The spin-polarized beams of short-lived radioactive isotopes are implanted into a crystal lattice. Quadrupole interaction is caused by the electric field gradient produced by the non-cubic crystal lattice surrounding the sodium nuclei [6]. The strength of the field gradient can be influenced by the choice of the host crystal, and it can thus be adapted to the size of the quadrupole moment to give a measurable interaction. The resolution of NMR measurements in solid crystals is in the $\mathrm{kHz}$ range. This corresponds to quadrupole moments of about $1 \mathrm{mb}$ for crystals with large internal field gradients.

The detection of $\beta$-NMR signals relies on the observation of an asymmetry in the angular distribution of the $\beta$-decay of a polarized ensemble of nuclear spins. This asymmetry depends on the $\beta$-decay properties and on the degree of nuclear polarization. The dominant decay channels of the neutron-rich sodium isotopes are allowed Gamow-Teller transitions. From the mostly known branching ratios and final states rather favourable conditions can be expected. Optical pumping is very efficient for the strong D1 and D2 lines of neutral atomic sodium (Na I) which lie in the frequency range where cw dye lasers give their maximum output power.

The measurement of quadrupole moments of light nuclei provides a sensitive test of theoretical models. Large-basis shell-model calculations led to a consistent microscopic description of the structure of $s d$-shell nuclei [7]. These calculations proved to be of high predictive power for a variety of observables. Substantial elements of the model are the construction of an effective interaction based on the whole oscillator shell and the diagonalization of the Hamiltonian to determine the eigenstates [8]. However, the exclusion of core configurations ( $1 s$ and the $1 p$ ) and of excitations to higher shells has to be accounted for by a renormalization of the operators. In the electric quadrupole operator this is done by introducing effective charges for the proton and the neutron [7,9]. These effective values are empirically deduced from E2 matrix elements mostly of stable nuclei. Experimental data on quadrupole moments far from stability are required to investigate whether these parameters also give an adequate description for nuclei with extreme $N / Z$ ratios.

Besides testing the scope of theoretical approaches, the present measurements of the 
quadrupole moments of ${ }^{26-29} \mathrm{Na}$ give insight into the development of nuclear structure and collective properties when approaching $N=20$, where a region of nuclear deformation is assumed to be centered around ${ }^{31} \mathrm{Na}[10,11]$. No indication of deformation was found from $\beta$-decay and binding energy studies for the isotopes with $N \leq 18$ [12]. For sodium the onset of deformation related to configuration mixing with $f p$-shell states is expected at $N=$ $19[13,11]$. Recent measurements of the quadrupole moments of ${ }^{30} \mathrm{Na}$ and ${ }^{31} \mathrm{Na}$, using a modified experimental procedure and a different host crystal lattice are being analyzed and will be reported separately [14]. Here we give improved values of the magnetic moments of ${ }^{27-31} \mathrm{Na}$ showing that the $s d$-shell predictions for $N=19$ and 20 are relatively poor. For ${ }^{31} \mathrm{Na}$ we also confirm the spin assignment $I=3 / 2$ [15] which is in contradiction to an sd-shell closure.

\section{$2 \quad$ Experiment}

\subsection{Experimental setup and procedures}

The experiments were performed at the ISOLDE on-line mass separator which is connected to the PS-Booster synchrotron at CERN. Neutron-rich sodium isotopes were produced by fragmentation of uranium in a $\mathrm{UC}_{2}$-target exposed to the $1 \mathrm{GeV}$ pulsed proton beam. Pulses of 2 to $3 \times 10^{13}$ protons with a duration of $2.4 \mu$ s were impinging on the target every $3.6 \mathrm{~s}$. After ionization to the charge state +1 in a hot tungsten tube, the product isotopes were electrostatically extracted and accelerated to an energy of $60 \mathrm{keV}$. The half-lives and typical production yields are given in Table 1 . The number of atoms per $\mu \mathrm{C}$ of proton beam roughly corresponds to the average number of atoms per second.

A schematic view of the apparatus is shown in Figure 1. The selected mass-separated ion beam is superimposed collinearly on the circularly polarized light beam of a cw dye laser. Optical excitation in the D1 or D2 line $\left({ }^{2} \mathrm{~S}_{1 / 2} \rightarrow{ }^{2} \mathrm{P}_{1 / 2,3 / 2}\right.$ at $\left.589 \mathrm{~nm}\right)$ of $\mathrm{Na} \mathrm{I}$ is performed after neutralizing the ions in a charge-transfer reaction [16]. For this purpose the beam passes through a cell containing sodium vapour at a density of about $2 \times 10^{14}$ atoms $/ \mathrm{cm}^{2}$. A variable electrical potential applied to that cell is used to tune the beam velocity and correspondingly the Doppler shift of the optical transition. This offers an easy way of tuning the laser frequency to the optical resonance.

For optical pumping the circularly polarized light interacts with the atomic beam over a path length of $1.5 \mathrm{~m}$. A weak longitudinal magnetic field of $1 \mathrm{mT}$ over this region, provided by a solenoidal coil, defines the polarization axis. Transverse field components disturbing the optical pumping process are suppressed by a $\mu$-metal shield around the beam tube. More details about the conditions of polarizing sodium atoms by narrowband laser light will be given in Section 3 .

The NMR setup implies the application of a strong magnetic field perpendicular to the beam axis and thus a rotation of the polarization axis by $90^{\circ}$. This is performed at the entrance of the NMR magnet, where the stray field in combination with an appropriate configuration of longitudinal fields ensures an adiabatic rotation of the spins, with a minimum loss of polarization. This rotational field does not exceed the strength of about $50 \mathrm{mT}$, in order to avoid decoupling of the small nuclear magnetic moment from the electronic magnetic moment.

After complete spin rotation the decoupling takes place in the strong transverse field. The radioactive atoms are implanted into a crystal which is placed in the center of the NMR magnet producing a homogeneous magnetic field in the range of 0.2 to 0.5 T. The $\beta$-decay asymmetry which is proportional to the degree of nuclear polarization is measured by two detector telescopes, each consisting of two plastic scintillators of 2 
mm thickness and covering a solid angle of $9 \%$ of $4 \pi$. These are placed between the thin windows of the vacuum chamber and the magnet pole faces.

For NMR measurements the host crystal is exposed to a magnetic radio frequency (rf) field produced with the help of two coils in Helmholtz configuration. These rf coils are fed by a programmable synthesizer in combination with a $100 \mathrm{~W}$ broad-band amplifier which is coupled to the coils by a resonant circuit. At the typical frequency of $2 \mathrm{MHz}, \mathrm{rf}$ amplitudes up to $0.5 \mathrm{mT}$ can be produced at the site of the crystal.

The optical excitation frequency is tuned to the atomic hyperfine structure component that gives the maximum $\beta$-asymmetry signal. In order to avoid strong fluctuations in the count rates, the measuring cycle is synchronized with the proton pulse structure of PS booster. The data acquisition of each channel (corresponding to a step in the rf) is triggered by the impact of a proton pulse on the production target. A beam of radioactive atoms is transmitted to the NMR setup during about 3 half-lives, and the implanted sample is irradiated while the $\beta$-asymmetry is detected. Then the $\mathrm{rf}$ is stepped to the next value before the next proton pulse arrives.

\section{$2.2 \beta$-NMR}

In a static magnetic field the energy of the nuclear spin system is split into $2 I+1$ Zeeman levels. If an additional rf field is in resonance with the splitting between adjacent Zeeman levels, magnetic dipole transitions are induced. This means that the degree of nuclear polarization is reduced, leading to a reduction of the observed $\beta$-decay asymmetry.

For host crystal lattice surroundings of cubic symmetry, the Zeeman levels are equidistant. Thus they are all coupled at one frequency which in saturation destroys the polarization completely. With the known magnetic field $\vec{B}$ this Larmor frequency $\nu_{L}$ yields the nuclear $g$-factor from the relation

$$
\nu_{L}=g_{I} \mu_{N}|\vec{B}| / h
$$

The additional interaction with an electric field gradient $V_{z z}$ present at well defined sites in non-cubic lattices can be used for measuring electric quadrupole moments [6]. An axially symmetric field gradient shifts the energy of the Zeeman levels of a nucleus with spin $I$ and quadrupole moment $Q$ according to

$$
E\left(m_{I}\right)=-h \nu_{L} m_{I}+\frac{e Q V_{z z}}{4 I(2 I-1)} \frac{1}{2}\left(3 \cos ^{2} \theta-1\right)\left(3 m_{I}^{2}-I(I+1)\right),
$$

where the angle $\theta$ describes the orientation of the internal electric field gradient with respect to the magnetic field.

The quadrupole interaction results in an equidistant splitting of the NMR signal into $2 \times I$ components. This splitting is proportional to the nuclear moment $Q$ and to the electric field gradient $V_{z z}$ at the site of the nucleus. The relative amplitudes of the resonances are essentially given by the distribution of the population over the Zeeman levels. If this distribution is known, an assignment of the levels which are coupled by the rf can be deduced from the line intensities in the spectrum. In this way the sign of the quadrupole interaction, i.e. of the product of the field gradient and the quadrupole moment, can be determined.

\section{$3 \quad$ Optical pumping}

The optical pumping conditions will be discussed taking as an example the excitation in the D2 line ${ }^{2} \mathrm{~S}_{1 / 2} \rightarrow{ }^{2} \mathrm{P}_{3 / 2}$ which was used for most of the measurements. In the weak 
longitudinal magnetic field the excitation with circularly polarized laser light, for instance $\sigma^{+}$-light, obeys the selection rules $\Delta F=0, \pm 1$ and $\Delta m_{F}=+1$, whereas spontaneous decay of the excited state is allowed for all channels with $\Delta F=0, \pm 1$ and $\Delta m_{F}=0, \pm 1$. Thus after several cycles of excitation and spontaneous emission the population is accumulated in the state with the maximum $m_{F}$ quantum number. This corresponds to an orientation of the angular momentum $F$ which is composed of the electronic and the nuclear spins.

Since the excitation is performed in collinear geometry, the Doppler broadening of the optical transition is reduced to a residual value of about $30 \mathrm{MHz}$ [16]. This inhomogeneous width is fully covered by the homogeneous power broadening of 50 to $100 \mathrm{MHz}$ for a power density of nearly $0.5 \mathrm{~W} / \mathrm{cm}^{2}$ used for efficient optical pumping.

The given conditions involve certain limitations for the maximum polarization that can be achieved. All investigated isotopes have a well resolved hyperfine structure splitting of the ${ }^{2} \mathrm{~S}_{1 / 2}$ ground state in the range of 1.5 to $3 \mathrm{GHz}$. Thus, only the fraction of atoms populating one of the two hyperfine structure levels is involved in the optical pumping process.

Furthermore, the splitting of the excited ${ }^{2} \mathrm{P}_{3 / 2}$ state remains partly unresolved. Thus the optical excitation is not restricted to the desirable closed system $\left|{ }^{2} \mathrm{~S}_{1 / 2} ; F=I+1 / 2\right\rangle \leftrightarrow$ $\left|{ }^{2} \mathrm{P}_{3 / 2} ; F^{\prime}=I+3 / 2\right\rangle$. As a consequence, the population of the $\left|{ }^{2} \mathrm{~S}_{1 / 2} ; F=I+1 / 2\right\rangle$ state is pumped after a few cycles of excitation and reemission via the nearby hyperfine structure levels of ${ }^{2} \mathrm{P}_{3 / 2}$ to the $F=I-1 / 2$ component of the ground state, and the optical pumping process is terminated.

Additional losses of polarization occur while the direction of the angular momenta is rotated into the transverse field of the NMR magnet. Since here the magnetic field is not parallel to the direction of propagation of the laser light, the excitation does no longer obey the $\Delta m_{F}=+1$ selection rule and the polarization is reduced. This depolarization process is suppressed for $\sigma^{+}$pumping on the transition $\left|{ }^{2} \mathrm{~S}_{1 / 2} ; F=I+1 / 2\right\rangle \leftrightarrow \mid{ }^{2} \mathrm{P}_{3 / 2} ; F^{\prime}=$ $I+3 / 2\rangle$, where the increasing magnetic field shifts most of the Zeeman components out of resonance.

The experimental spectrum in Figure 2 shows the $\beta$-asymmetry as a function of the optical excitation frequency for the $I=3$ isotope ${ }^{26} \mathrm{Na}$. The two frequency positions giving nuclear polarization signals correspond to excitations from the two hyperfine structure components of the ground state. The negative tail of the higher-frequency signal is the result of optical pumping in the non-longitudinal field region.

As expected the highest degree of polarization is obtained at the frequency of the transition $\left|{ }^{2} \mathrm{~S}_{1 / 2} ; F=I+1 / 2\right\rangle \leftrightarrow\left|{ }^{2} \mathrm{P}_{3 / 2} ; F^{\prime}=I+3 / 2\right\rangle$. The corresponding maximum values of the experimental $\beta$-decay asymmetries are compiled in Table 1 for the isotopes ${ }^{26-31} \mathrm{Na}$. Taking into account the known $\beta$-decay properties of ${ }^{26} \mathrm{Na},{ }^{27} \mathrm{Na}$ and ${ }^{28} \mathrm{Na}$ one finds that polarizations between 0.4 and 0.6 are reached.

In order to understand the measured frequency and power dependences of the polarization, one has to calculate the distribution of population over the Zeeman levels. This distribution also directly affects the relative intensities of the lines in the NMR spectrum. A simulation of the optical pumping process has been performed by solving a system of rate equations,

$$
\frac{d N_{i}}{d t}=\sum_{j} C_{i j}\left(\nu_{i j}, \nu_{\text {Laser }}\right)\left(N_{j}^{\prime}-N_{i}\right)+\sum_{j} A_{i j} N_{j}^{\prime}
$$




$$
\frac{d N_{j}^{\prime}}{d t}=\sum_{i} C_{i j}\left(\nu_{i j}, \nu_{\text {Laser }}\right)\left(N_{i}-N_{j}^{\prime}\right)-\sum_{i} A_{i j} N_{j}^{\prime}
$$

where $N_{i}$ and $N_{j}^{\prime}$ denote the relative populations of the Zeeman sublevels of the ${ }^{2} \mathrm{~S}_{1 / 2}$ and the ${ }^{2} \mathrm{P}_{3 / 2}$ state, respectively. They are interrelated by the probabilities for induced absorption and emission $C_{i j}\left(\nu_{i j}, \nu_{\text {Laser }}\right)$ and for spontaneous emission $A_{i j}$. This system of coupled differential equations is solved numerically, yielding the population of each level after the interaction with the laser light. Losses in the non-longitudinal field region are taken into account by assuming a small fraction of the laser radiation with opposite polarization. The result of such a calculation, included in Figure 2, describes all essential features of the experimental spectrum.

Table 1: Half-lives, yields and experimental $\beta$-decay asymmetries for the isotopes ${ }^{26-31} \mathrm{Na}$. The asymmetry values measured in a NaF host crystal are corrected for relaxation losses during the lifetime and for the solid angle of the detector.

\begin{tabular}{|c||c|c|c|c|c|c|}
\hline Isotope & ${ }^{26} \mathrm{Na}$ & ${ }^{27} \mathrm{Na}$ & ${ }^{28} \mathrm{Na}$ & ${ }^{29} \mathrm{Na}$ & ${ }^{30} \mathrm{Na}$ & ${ }^{31} \mathrm{Na}$ \\
\hline$T_{1 / 2}$ & $1.07 \mathrm{~s}$ & $301 \mathrm{~ms}$ & $30.5 \mathrm{~ms}$ & $44.9 \mathrm{~ms}$ & $48 \mathrm{~ms}$ & $17 \mathrm{~ms}$ \\
Yield $/ \mu \mathrm{C}$ & $3.2 \times 10^{7}$ & $9 \times 10^{6}$ & $5.7 \times 10^{5}$ & $1.5 \times 10^{5}$ & $2 \times 10^{4}$ & $4 \times 10^{2}$ \\
Asymmetry & $-0.366(12)$ & $-0.350(10)$ & $-0.442(7)$ & $-0.225(23)$ & $-0.19(3)$ & $-0.25(5)$ \\
\hline
\end{tabular}

The inset of Figure 2 shows the population of the nuclear Zeeman levels after the adiabatic decoupling of nuclear and electronic spins in the transition to the strong magnetic field. This distribution has been calculated for optical pumping in the asymmetry maximum, which corresponds to the conditions employed for the NMR experiment. A strong accumulation in the $m_{I}=I$ state and a flattening towards smaller and negative values of the $m_{I}$ quantum number is characteristic for this distribution.

The differences of the populations of neighbouring nuclear Zeeman levels give the relative amplitudes of the lines in the NMR spectra. For the described $\sigma^{+}$optical pumping (from the $F=I+1 / 2$ ground state component) the strongest NMR line is the one connecting the $m_{I}=I$ and $m_{I}=I-1$ levels. Changing to $\sigma^{-}$optical pumping from the $F=I-1 / 2$ ground state component essentially leads to opposite conditions with the resonance of largest amplitude connecting $m_{I}=-I$ and $m_{I}=-(I-1)$. This behaviour is exploited for extracting the sign of the quadrupole interaction (cf. Section 2.2).

\section{$4 \quad$ Experimental results}

\section{1 $g$-factors}

The magnetic moments of the isotopes ${ }^{20-31} \mathrm{Na}$ have been known from laser spectroscopy measurements of the ground state hyperfine structure [15, 1]. Even though it was not the aim of the present experiment to measure nuclear $g$-factors, these results could be improved for the isotopes ${ }^{27-31} \mathrm{Na}$.

The nuclear Larmor frequencies were determined by $\beta$-NMR measurements in the cubic host lattice of a NaF single crystal or a gold foil. During these measurements the magnetic field was kept constant. As the absolute strength of this field is not accurately known, only the ratios of the Larmor frequencies can be used to determine relative $g$ factors. Absolute values are then derived using the value $\mu_{I}\left({ }^{26} \mathrm{Na}\right)=2.851(2) \mu_{N}$ from ref. [15]. The results are listed in Table 2 together with the literature values. 
The consistency of the magnetic moments of ${ }^{31} \mathrm{Na}$ derived from atomic hyperfine structure [15] and from the present direct $g$-factor measurement confirms that the spin assumption of ref. [15], $I\left({ }^{31} \mathrm{Na}\right)=3 / 2$, is correct.

Table 2: Magnetic moments and $g$-factor ratios for ${ }^{26-31} \mathrm{Na}$. The results of $s d$-shell calculations are given for comparison. Literature values are taken from ref. $[15,1]$

\begin{tabular}{|c|c||c|c|c||c|c|}
\hline Isotope & $I$ & $g_{I} / g_{I}\left({ }^{26} \mathrm{Na}\right)$ & \multicolumn{2}{|c||}{$\mu_{I}(\exp ) / \mu_{N}$} & \multicolumn{2}{|c|}{$\mu_{I}$ (theory) $/ \mu_{N}$} \\
& & & this work & ref. [1, 15] & sd-free & $s d$-eff \\
\hline${ }^{26} \mathrm{Na}$ & 3 & - & - & $2.851(2)^{*}$ & 2.722 & 2.780 \\
${ }^{27} \mathrm{Na}$ & $5 / 2$ & $1.63914(10)$ & $3.894(3)$ & $3.895(5)$ & 3.711 & 3.773 \\
${ }^{28} \mathrm{Na}$ & 1 & $2.54627(20)$ & $2.420(2)$ & $2.426(5)$ & 2.254 & 2.316 \\
${ }^{29} \mathrm{Na}$ & $3 / 2$ & $1.72342(12)$ & $2.457(2)$ & $2.449(8)$ & 2.455 & 2.468 \\
${ }^{30} \mathrm{Na}$ & 2 & $1.0887(6)$ & $2.069(2)$ & $2.083(10)$ & 2.554 & 2.687 \\
${ }^{31} \mathrm{Na}$ & $3 / 2^{a}$ & $1.61206(12)$ & $2.298(2)$ & $2.305(9)$ & 2.655 & 2.673 \\
\hline
\end{tabular}

* Reference value

${ }^{a}$ From the $s d$-shell calculation this is not the ground state

\subsection{Quadrupole moments}

\subsection{1 $\mathrm{LiNbO}_{3}$ results}

The measurement of relative quadrupole moments for the series of isotopes ${ }^{26-29} \mathrm{Na}$ has been performed in a $\mathrm{LiNbO}_{3}$ lattice. In this lattice the implanted sodium nuclei experience a comparatively large electric field gradient. This is important for the measurement of the extremely small quadrupole moments of ${ }^{26} \mathrm{Na}$ and ${ }^{27} \mathrm{Na}$. Furthermore, the $\beta$-decay asymmetries are essentially as large as observed for the cubic NaF lattice.

Figure 3 shows the NMR spectra for ${ }^{26} \mathrm{Na}(I=3),{ }^{27} \mathrm{Na}(I=5 / 2),{ }^{28} \mathrm{Na}(I=1)$ and ${ }^{29} \mathrm{Na}(I=3 / 2)$ in $\mathrm{LiNbO}_{3}$, obtained for optical pumping with $\sigma^{+}$light. As a consequence of the non-linear distribution of population over the Zeeman levels not all resonances have sufficiently large amplitudes to be clearly visible in the spectra. For the isotopes with small quadrupole moments and larger spin, ${ }^{26} \mathrm{Na}$ and ${ }^{27} \mathrm{Na}$, the resonances are only partly resolved and only the higher-frequency components with $\nu \geq \nu_{L}$ are shown. In the spectrum of ${ }^{29} \mathrm{Na}$ the weak line at $5.5 \mathrm{MHz}$ was enhanced by changing to $\sigma^{-}$optical pumping from the $F=I-1 / 2$ ground state component $\left(\Delta m_{F}=-1\right)$. This gives opposite polarization as shown at the right-hand axis of the plot.

With the optical pumping scheme described in Section 3 (excitation from the $F=$ $I+1 / 2$ ground state component with selection rule $\Delta m_{F}=+1$ ) the slope in the amplitudes of the resonances of ${ }^{26} \mathrm{Na}$ and ${ }^{27} \mathrm{Na}$ on the one hand, and of ${ }^{28} \mathrm{Na}$ and ${ }^{29} \mathrm{Na}$ on the other hand show that the quadrupole interaction energies have different sign. Since the electric field gradients are isotope-independent, this means that the quadrupole moments have opposite sign.

Extremely large line widths of 200 to $300 \mathrm{kHz}$ are observed in the spectra of ${ }^{28} \mathrm{Na}$ and ${ }^{29} \mathrm{Na}$ for all resonances whose frequency position is affected by the large quadrupole interaction. Furthermore, these lines exhibit a double-peak structure which is well visible only in the case of ${ }^{29} \mathrm{Na}$ where the splitting is largest. In contrast to this, the resonance at the Larmor frequency (for ${ }^{29} \mathrm{Na}$ ) remains narrow and does not show any substructure. 
These line shapes can be explained by the interaction of the nuclear quadrupole moment with a continuous distribution of electric field gradients caused by radiation damage of the lattice. The presence of two discrete field gradients is well known from PAC studies [17] of ${ }^{111} \mathrm{In}$ in $\mathrm{LiNbO}_{3}$ and it has been attributed to the occupation of lithium sites either with a perfect lattice environment or a defect in the vicinity. Therefore, the analysis of the spectra is based on the interaction of the quadrupole moment with two different field gradients. Their relative strength as well as the population of the respective lattice sites is considered to be the same for all isotopes. Two Gaussians with fixed relative amplitudes and widths are used to parametrize the resonance structures which are shifted by the quadrupole interaction. The line at the Larmor frequency (only existing for isotopes with half-integer spin) is well described by a single Gaussian.

Another small fraction of atoms is situated on sites for which a distribution of small field gradients centered around zero has to be assumed. This results in a resonance structure around the Larmor frequency which is observed for the $I=3$ isotope ${ }^{26} \mathrm{Na}$. For the other isotopes this structure either coincides with the regular line at $\nu_{L}\left({ }^{27} \mathrm{Na}\right)$, or the inhomogeneous line width scaling with the quadrupole moment becomes so large that the line is smeared out $\left({ }^{28} \mathrm{Na}\right.$ and $\left.{ }^{29} \mathrm{Na}\right)$. In these latter cases it is not explicitly taken into account in the fitting procedure. The quadrupole interaction constants and their relative signs obtained from $\chi^{2}$-fits with a consistent parametrization of the spectra are compiled in Table 3.

\subsubsection{Calibration in $\mathrm{NaNO}_{3}$}

A calibration of the absolute values of the quadrupole moments has been performed by measuring the quadrupole splitting of ${ }^{28} \mathrm{Na}(I=1)$ in the lattice of $\mathrm{NaNO}_{3}$, for which the electric field gradient at the substitutional sodium site is known [6]. This lattice is not suitable for $\beta$-NMR measurements on the whole series of isotopes, because the experimental asymmetries are five times lower than in the $\mathrm{LiNbO}_{3}$ host lattice, and because the field gradient is too small to resolve the quadrupole interaction of ${ }^{26} \mathrm{Na}$ and ${ }^{27} \mathrm{Na}$.

The spectrum is displayed in Figure 4. As in the case of $\mathrm{LiNbO}_{3}$ a small fraction of nuclei populating lattice sites with randomly distributed small field gradients is observed, giving rise to a broad resonance at the Larmor frequency. A Gaussian fit based on this assumption yields a quadrupole interaction constant $\nu_{Q}\left({ }^{28} \mathrm{Na}\right)=\left|(e / h) V_{z z} Q\left({ }^{28} \mathrm{Na}\right)\right|=$ $124.8(3.4) \mathrm{kHz}$.

This interaction constant of ${ }^{28} \mathrm{Na}$ in $\mathrm{NaNO}_{3}$ can be ascribed to regular lattice sites for which also the quadrupole interaction of ${ }^{23} \mathrm{Na}$ is known [6]. This is not only expected from general experience but also supported by experimental arguments. Only two sharp resonances are visible in the spectrum of Figure 4, and their depths corresponds to the destruction most of the asymmetry.

\subsection{Determination of quadrupole moments}

The quadrupole interaction constant for stable ${ }^{23} \mathrm{Na}$ in $\mathrm{NaNO}_{3}$ has been measured by classical NMR spectroscopy to be $334(2) \mathrm{kHz}$ [6]. Using this reference value and the quadrupole moment of ${ }^{23} \mathrm{Na}$, one can derive the absolute value of the quadrupole moment of ${ }^{28} \mathrm{Na}$. Absolute values of the moments of ${ }^{26-29} \mathrm{Na}$ are then determined by the ratios of the interaction constants measured in the $\mathrm{LiNbO}_{3}$ crystal.

For the reference quadrupole moment of ${ }^{23} \mathrm{Na}$ precise but partly incompatible results exist in the literature. Muonic X-ray spectroscopy [18] yielded $Q\left({ }^{23} \mathrm{Na}\right)_{\mu}=100.6(2.0) \mathrm{mb}$. However, the accuracy of this value was questioned when atomic structure calculations 
Table 3: Experimental quadrupole interaction constants in $\mathrm{LiNbO}_{3}$ and $\mathrm{NaNO}_{3}$.

\begin{tabular}{|c|c|c|c|}
\hline Isotope & $\begin{array}{c}\nu_{Q}\left(\mathrm{LiNbO}_{3}\right) \\
\mathrm{kHz}\end{array}$ & $\begin{array}{c}\nu_{Q}\left(\mathrm{NaNO}_{3}\right) \\
\mathrm{kHz}\end{array}$ & $\operatorname{sign}(A / \operatorname{sign}(28))$ \\
\hline${ }^{23} \mathrm{Na}$ & & $334(2)^{a}$ & \\
\hline${ }^{26} \mathrm{Na}$ & $144.3(3.5)$ & & - \\
\hline${ }^{27} \mathrm{Na}$ & $196.2(2.9)$ & & - \\
\hline${ }^{28} \mathrm{Na}$ & $1077(4)$ & $124.8(3.4)$ & ref. \\
\hline${ }^{29} \mathrm{Na}$ & $2334(13)$ & & + \\
\hline
\end{tabular}

${ }^{a}$ Reference [6]

$[19,20,21]$ of electric field gradients reached the $1 \%$ accuracy level. Combined with atomic hyperfine structure measurements of the quadrupole coupling constant ( $B$-factor) of the ${ }^{2} \mathrm{P}_{3 / 2}$ level [22, 23] several independent calculations yield quadrupole moments that are $4 \%-6 \%$ larger. These values are supported by an analysis of the quadrupole interaction in $\mathrm{NaF}$ and $\mathrm{NaCl}$ molecules, yielding 104(1.2) mb [24], and by nuclear-model-dependent values from Coulomb excitation measurements [25, 18].

The moments compiled in Table 4 and displayed in Figure 5 have been calculated using $Q\left({ }^{23} \mathrm{Na}\right)=105.6(1.2) \mathrm{mb}$ from the most accurate measurement of the $B$-factor [23] and the recent most complete multi-configuration Hartree-Fock calculation [21]. Since only relative signs for the quadrupole moments of ${ }^{26-29} \mathrm{Na}$ have been determined experimentally, the missing information about at least one sign has been replaced by the overall expectation from the shell-model (see Section 5.2).

\section{Discussion}

\subsection{Comparison to previous results}

Quadrupole moments of ${ }^{26-29} \mathrm{Na}$ were already reported from laser-rf double resonance measurements of the hyperfine structure of the ${ }^{2} \mathrm{P}_{3 / 2}$ state [1]. The atoms were excited by laser light to the $F=I+3 / 2$ component, and an rf field was applied to induce transitions between $F=I+3 / 2$ and $F=I+1 / 2$ leading to a change in atomic polarization which served for resonance detection. The accuracy of about $1 \mathrm{MHz}$ achieved in the determination of frequency intervals was essentially given by a systematic error accounting for a slightly off-resonant frequency of the laser. Considering the natural line width of $10 \mathrm{MHz}$ this experimental technique nearly reaches the ultimate precision of optical spectroscopy on short-lived isotopes. We have recalculated the reported quadrupole moments [1] from the originally measured hyperfine structure splittings using a recent more accurate reference value for the $A\left({ }^{2} \mathrm{P}_{3 / 2}\right)$ factor of ${ }^{23} \mathrm{Na}$ [23] and the new calibration value for $Q\left({ }^{23} \mathrm{Na}\right)$. These results are included in Table 4 and in Figure 5.

Our reevaluation somewhat reduces the nearly constant difference of about $-50 \mathrm{mb}$ between the original data of ref. [1] and the present results. As shown in Figure 5 the remaining systematic deviation of the hyperfine structure results towards more negative quadrupole moments is now just compatible with the estimated experimental errors.

\subsection{Shell-model calculations}

The development of large-basis shell-model calculations has greatly improved the description of the structure of light nuclei in the $p$ and $s d$ shells [7]. In particular, the electromagnetic nuclear moments can be reproduced or predicted rather precisely. Still, 
the agreement between theory and experiment is less quantitative for the quadrupole moment than for the magnetic moments. Quadrupole moments are more sensitive to core excitations and are thus more difficult to describe theoretically in a necessarily restricted model space. On the other hand, there are problems to deduce very accurate values of quadrupole moments from experimental hyperfine structure data. These data partly suffer from uncertainties in the knowledge of atomic hyperfine field given by the electron wave function. Only for a few cases the electric field gradient can be calculated as reliably as for sodium.

The isotopes ${ }^{26-29} \mathrm{Na}$ are situated in the $s d$ shell between the regions of nuclear deformation centered around ${ }^{23} \mathrm{Na}$ and ${ }^{31} \mathrm{Na}$. The calculation of the moments is based upon the wave functions obtained in the full $s d$ model space $\left(1 d_{5 / 2}, 1 d_{3 / 2}, 2 s_{1 / 2}\right)$ with the USD Hamiltonian of Brown and Wildenthal [7]. These calculations with the $s d$-shell basis can account for the deformation near ${ }^{23} \mathrm{Na}$, but the $s d$-shell wave functions for ${ }^{31} \mathrm{Na}$ and other nuclei with $N=20$ such as ${ }^{32} \mathrm{Mg}$ are spherical. To account for the deformation near $N=20$ one has expand the basis to include the $f p$-shell intruder states. But for the comparison to the results in this work we stay to the $s d$-shell basis. The main focus of this discussion will be on the quadrupole moments. However, for completeness we give in Table 2 the USD results for the magnetic moments obtained with the free-nucleon M1 operator (sd-free) and with the effective M1 operator [26] (sd-eff). Up to ${ }^{29} \mathrm{Na}$ the comparison with experiment and theory is good, but becomes significantly worse for ${ }^{30} \mathrm{Na}$ and ${ }^{31} \mathrm{Na}$. This is probably a indication of the $f p$-shell intruder nature of these states.

Table 4: Comparison of the quadrupole moments to former results Ref. [1] and to shellmodel calculations assuming different values for the effective charges $e_{p}$ and $e_{n}$ and different radial wave functions $\rho$.

\begin{tabular}{|c|c|c|c|c|c|c|c|c|c|}
\hline & $J$ & $T$ & \multicolumn{2}{|c|}{$Q(\exp )[\mathrm{mb}]$} & \multicolumn{5}{|c|}{$Q($ theory $)[\mathrm{mb}]$} \\
\hline & & & $\beta$-NMR & Lit. & $\begin{array}{l}e_{p} \\
e_{n} \\
\rho\end{array}$ & $\begin{array}{c}1 \\
0 \\
\mathrm{HO}\end{array}$ & $\begin{array}{c}1.35 \\
0.35 \\
\mathrm{HO}\end{array}$ & $\begin{array}{l}1.25 \\
0.45 \\
\mathrm{HO}\end{array}$ & $\begin{array}{l}1.35 \\
0.35 \\
\text { SGII }\end{array}$ \\
\hline${ }^{23} \mathrm{Na}$ & $3 / 2+$ & $1 / 2$ & & $+105.6(1.2)^{a}$ & & 59.9 & 103.4 & 103.8 & 110.8 \\
\hline${ }^{24} \mathrm{Na}$ & $4+$ & 1 & & & & 148.1 & 243.2 & 240.8 & 253.6 \\
\hline${ }^{25} \mathrm{Na}$ & $5 / 2+$ & $3 / 2$ & & $-64(44)^{b}$ & & -6.4 & -4.6 & -2.9 & -4.4 \\
\hline${ }^{26} \mathrm{Na}$ & $3+$ & 2 & $-5.3(2)$ & $-56(47)^{b}$ & & -8.0 & -11.4 & -10.8 & -11.7 \\
\hline${ }^{27} \mathrm{Na}$ & $5 / 2+$ & $5 / 2$ & $-7.2(3)$ & $-29(45)^{b}$ & & -4.6 & -12.5 & -13.8 & -12.9 \\
\hline${ }^{28} \mathrm{Na}$ & $1+$ & 3 & $+39.5(1.2)$ & $-4(31)^{b}$ & & 26.9 & 40.6 & 39.1 & 40.5 \\
\hline${ }^{29} \mathrm{Na}$ & $3 / 2+$ & $7 / 2$ & $+86(3)$ & $+38(47)^{b}$ & & 47.8 & 67.9 & 64.0 & 66.9 \\
\hline${ }^{30} \mathrm{Na}$ & $2+$ & 4 & & & & -67.0 & -100.0 & -96.0 & -98.9 \\
\hline${ }^{31} \mathrm{Na}$ & $3 / 2+{ }^{c}$ & $9 / 2$ & & & & 41.6 & 56.2 & 52.0 & 54.3 \\
\hline
\end{tabular}

${ }^{a}$ Refs. [23, 21], evaluation see text.

$b$ The results quoted in ref. [1] have been recalculated following the original procedure and using more recent reference values for the hyperfine structure of ${ }^{23} \mathrm{Na}$.

${ }^{c}$ From $s d$-shell calculation the $3 / 2^{+}$state is $454 \mathrm{keV}$ above the $5 / 2^{+}$ground state.

In Table 4 the experimental results for the quadrupole moments are compared to results of the shell-model calculations. The USD wave functions have been used to calculate 
a variety of observables in the $s d$ shell related to the quadrupole operator, in particular the quadrupole moments which were known in 1986 [9] and the quadrupole moments of neutron-deficient argon isotopes [27]. One needs to combine the multiparticle information contained in the $s d$-shell wave functions with the single-particle matrix elements [9]. For the single-particle radial wave functions we use those obtained with the simple harmonic oscillator (HO) with $\hbar \omega=45 A^{-1 / 3}-25 A^{-2 / 3}$, and those obtained with the more realistic spherical Hartree-Fock calculation using the SGII Skyrme interaction [28]. These SGII radial wave functions were used recently for calculations of the matter and charge radii of the $\mathrm{Na}$ isotopes [29].

The results obtained with the HO radial wave functions together with the freenucleon charges of $e_{p}^{\text {free }}=1$ for the proton and $e_{n}^{\text {free }}=0$ for the neutron are given in the first theoretical column of Table 4. One notices here as well as for all other quadrupole observables in the $s d$ shell that the experimental matrix elements are systematically larger than those calculated. The reason for this is the well known effect of the polarization of the core protons by the valence protons and neutrons [30,31]. This polarization represents part of the actual wave functions which is left out of the truncation to the $s d$ shell. Empirically it can be accounted for by replacing the free-nucleon charges with effective charges $e_{p}^{\text {eff }}=1+\delta e_{p}$ and $e_{n}^{\text {eff }}=0+\delta e_{n}$, where the $\delta e$ are the polarization charges.

The empirical isoscalar polarization charge is well determined and has a value in the range of $\delta e_{p}+\delta e_{n}=0.70$ [9] to 0.78 [7]. This range of empirical values for the isoscalar effective charge is in agreement with those calculated microscopically in perturbation theory [31]. The isovector polarization charge $\delta e_{p}-\delta e_{n}$ is more difficult to determine because most quadrupole data are dominated by the isoscalar component. All theoretical considerations $[30,31]$ result in $\delta e_{n}>\delta e_{p}$, because the valence neutrons are more effective in polarizing the core protons due to the stronger average proton-neutron interaction compared to the proton-proton interaction. An empirical value of about $\delta e_{n}-\delta e_{p}=0.2$ is consistent with the previous analysis of quadrupole moment and $B(\mathrm{E} 2)$ data $[9,7]$.

In order to test the sensitivity of sodium quadrupole moments to the value of the isovector effective charge we compare in Table 4 the purely isoscalar results obtained with $e_{p}=1.35$ and $e_{n}=0.35$ [9] with those obtained with an isovector polarization of $\delta e_{n}-\delta e_{p}=0.2$ which gives $e_{p}=1.25$ and $e_{n}=0.45$. One notices that the effect of adding an isovector polarization is small because the isovector component of the quadrupole moment is small relative to the isoscalar component.

Finally, we explore the sensitivity to the radial wave functions by repeating the isoscalar calculation with the SGII HF radial wave functions. The difference between the $\mathrm{HO}$ and HF results is small and does not improve the agreement between experiment and theory.

In general, the experimental results for ${ }^{23} \mathrm{Na}$ and ${ }^{28} \mathrm{Na}$ are well described by the $s d$ shell plus core-polarization calculations. The small quadrupole moments for ${ }^{26} \mathrm{Na}$ and ${ }^{27} \mathrm{Na}$ are also small in the calculations but their actual values are not so well reproduced. The absolute difference between experiment and theory for these small quadrupole moments is typical of the difference found for other quadrupole moments in the $s d$ shell [9]. The experimental quadrupole moment for ${ }^{29} \mathrm{Na}$ is somewhat larger than theory, perhaps indicating some influence from the $f p$-shell intruder state for this nucleus with $N=18$.

The small quadrupole moments of ${ }^{25-27} \mathrm{Na}$ are related to the fact that the main component $(37 \%)$ of the wave functions has the configuration of $\left(1 d_{5 / 2}\right)^{3}$ for protons and $\left(1 d_{5 / 2}\right)^{6},\left(2 s_{1 / 2}\right)^{0-2}$ for neutrons. For this main configuration, the neutrons have zero quadrupole moment because the valence neutrons outside the $1 d_{5 / 2}$ closed shell are in the 
$2 s$ state, and the protons also have a zero quadrupole moment because they are in the middle of a $j$ shell [32]. For the lighter and heavier isotopes, both protons and neutrons are in open shells, and the strong proton-neutron interaction gives rise to more collective (deformed) states. The experimental results from the present experiment nicely confirm the qualitative expectations of the simple shell model as well as the more quantitative predictions of the full $s d$-shell calculations.

\section{Conclusion}

The measurements on ${ }^{26-29} \mathrm{Na}$ presented in this paper show that $\beta$-NMR spectroscopy is a powerful method to investigate nuclear moments of light isotopes far from stability. This holds in particular for the nuclear quadrupole moments, because the strength of the quadrupole interaction can be influenced by the choice of the host lattice and very high resolution can be achieved.

Optical pumping to high degrees of nuclear polarization provides the sensitivity required for the investigation of weakly produced isotopes. Of course, this technique can only be applied to elements which have appropriate atomic spectra. Sodium, having strong optical resonance lines to the first excited states, turns out to be one of the best suited candidates for this method. In addition, the favourable $\beta$-decay properties of all investigated isotopes give high $\beta$-decay asymmetries.

Results of the present experiment comprise the quadrupole moments of isotopes up to mass number $A=29$. None of these measurements was limited by the production yields of the ISOLDE target, which are still about $10^{5}$ atoms per second for ${ }^{29} \mathrm{Na}$. The experimental difficulties arose from the requirement of finding host lattices with appropriate field gradients and relaxation properties. The measurement of the very small quadrupole moments of ${ }^{26} \mathrm{Na}$ and ${ }^{27} \mathrm{Na}$ on the one side, and the calibration with a known field gradient on the other were the major challenges.

The present investigation already includes the measurement of the decay asymmetries of ${ }^{30} \mathrm{Na}$ and ${ }^{31} \mathrm{Na}$. A measurement of the quadrupole moments [14] has to cope with much lower production yields of these isotopes and therefore needs some modification of the experimental procedure including a different choice of the host crystal. Data from such an experiment are being analyzed, yielding direct information about the development of nuclear structure in the $N=20$ region of postulated deformation.

We would like to thank B. Ittermann and J.G. Marques for helpful discussions on implantation sites in ion crystals. This work has been funded by the German Ministry for Education and Research (BMBF) under the Contracts No. 06 MZ 566 I and 06 MZ 866 I, and by the Belgian Fund for Scientific Research - Flanders (F.W.O.). B.A.B. acknowledges support from NSF grant PHY-9605207, from the British EPSRC and from the Alexander von Humboldt Foundation.

\section{References}

[1] F. Touchard, J.M. Serre, S. Büttgenbach, P. Guimbal, R. Klapisch, M. de Saint Simon, C. Thibault, H.T. Duong, P. Juncar, S. Liberman, J. Pinard and J.L. Vialle, Phys. Rev. C 25, 2756 (1982).

[2] H. Ackermann, P. Heitjans and H.J. Stöckmann in Hyperfine Interactions of Radioactive Nuclei, ed. J. Christiansen, Topics in Current Physics, Vol. 31 (Springer, Berlin, 1983), p. 291. 
[3] E. Arnold, J. Bonn, R. Gegenwart, W. Neu, R. Neugart, E.W. Otten, G. Ulm, K.Wendt and ISOLDE Collaboration, Phys. Lett. B 197, 311 (1987).

[4] E. Arnold, J. Bonn, W. Neu, R. Neugart, E.W. Otten and ISOLDE Collaboration, Z. Phys. A 331, 295 (1988).

[5] E. Arnold, J. Bonn, A. Klein, R. Neugart, M. Neuroth, E.W. Otten, P. Lievens, H. Reich, W. Widdra and ISOLDE Collaboration, Phys. Lett. B 281, 16 (1992).

[6] R.V. Pound, Phys. Rev. 79, 685 (1950).

[7] B.A. Brown and B.H. Wildenthal, Ann. Rev. Nucl. Part. Sci. 38, 29 (1988).

[8] B.H. Wildenthal, Prog. Part. Nucl. Phys. 11, 5 (1984).

[9] M. Carchidi, B.H. Wildenthal and B.A. Brown, Phys. Rev. C 34, 2290 (1986).

[10] X. Campi, H. Flocard, A.K. Kerman and S. Koonin, Nucl. Phys. A 251, 193 (1975).

[11] E.K. Warburton, J.A. Becker and B.A. Brown, Phys. Rev. C 41, 1147 (1990).

[12] B.H. Wildenthal, M.S. Curtin and B.A. Brown, Phys. Rev. C 28, 1343 (1983).

[13] E. Caurier, F. Nowacki, A. Poves and J. Retamosa, Phys. Rev. C 58, 2033 (1998).

[14] S. Wilbert, B.A. Brown, M. Keim, P. Lievens, R. Neugart, L. Vermeeren, to be published.

[15] G. Huber, F. Touchard, S. Büttgenbach, C. Thibault, R. Klapisch, H.T. Duong, S. Liberman, J. Pinard, J.L. Vialle, P. Juncar and P. Jacquinot, Phys. Rev. C 18, 2343 (1978).

[16] A.C. Mueller, F. Buchinger, W. Klempt, E.W. Otten, R. Neugart, C. Ekström and J. Heinemeier, Nucl. Phys. A 403, 234 (1983).

[17] B. Hauer, R. Vianden, J.G. Marques, N.P. Barradas, J.G. Correia, A.A. Melo, J.C. Soares, F. Agulló-López and E. Dieguez, Phys. Rev. B 51, 6208 (1995).

[18] B. Jeckelmann, W. Beer, I. Beltrami, F.W.N. de Boer, G. de Chambrier, P.F.A. Goudsmit, J. Kern, H.J. Leisi, W. Ruckstuhl and A. Vacchi, Nucl. Phys. A 408, 495 (1983).

[19] D. Sundholm and J. Olsen, Phys. Rev. Lett. 68, 927 (1992).

[20] S. Salomonson and A. Ynnerman, Phys. Rev. A 43, 88 (1991).

[21] P. Jönsson, A. Ynnerman, C. Froese Fischer, M.R. Godefroid and J. Olsen, Phys. Rev. A 53, 4021 (1996).

[22] T. Krist, P. Kuske, A. Gaupp, W. Wittmann and H.J. Andrä, Phys. Lett. A 61, 94 (1977).

[23] W. Yei, A. Sieradzan and M.D. Havey, Phys. Rev. A 48, 1909 (1993).

[24] P. Pyykkö and A.J. Sadlej, Chem. Phys. Lett. 227, 221 (1994).

[25] D. Schwalm, E.K. Warburton and J.W. Olness, Nucl. Phys. A 293, 425 (1977).

[26] B.A. Brown and B.H. Wildenthal, Nucl. Phys. A 474, 290 (1987).

[27] A. Klein, B.A. Brown, U. Georg, M. Keim, P. Lievens, R. Neugart, M. Neuroth, R.E. Silverans, L. Vermeeren and ISOLDE Collaboration, Nucl. Phys. A 607, 1 (1996).

[28] Nguyen Van Giai and H. Sagawa, Nucl. Phys. A 371, 1 (1981); Nguyen Van Giai and H. Sagawa, Phys. Lett. B 106, 379 (1981).

[29] B.A. Brown and W.A. Richter, Phys. Rev. C 54, 673 (1996).

[30] B.A. Brown, A. Arima and J.B. McGrory, Nucl. Phys. A 277, 77 (1977).

[31] H. Sagawa and B.A. Brown, Nucl. Phys. A 430, 84 (1984).

[32] A. de-Shalit and I. Talmi, Nuclear Shell Theory, (Academic Press, New York, 1963). 


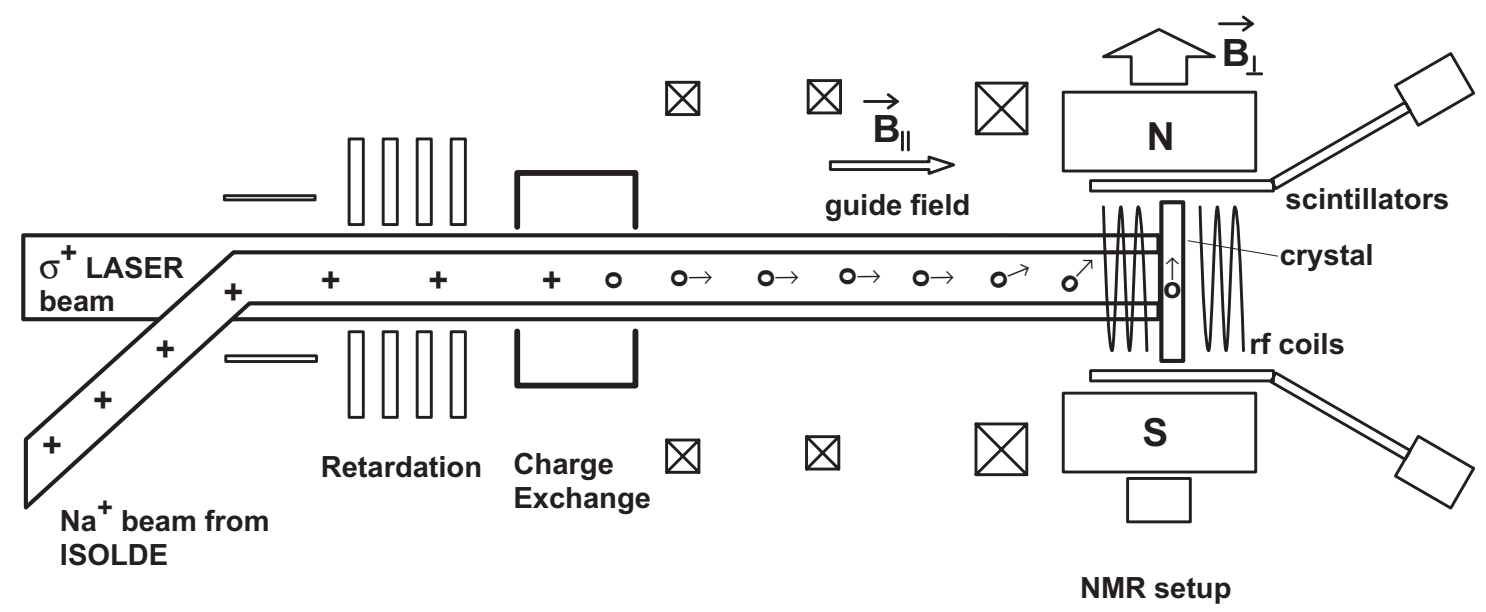

Figure 1: Experimental setup for in-beam optical pumping and $\beta$-NMR.

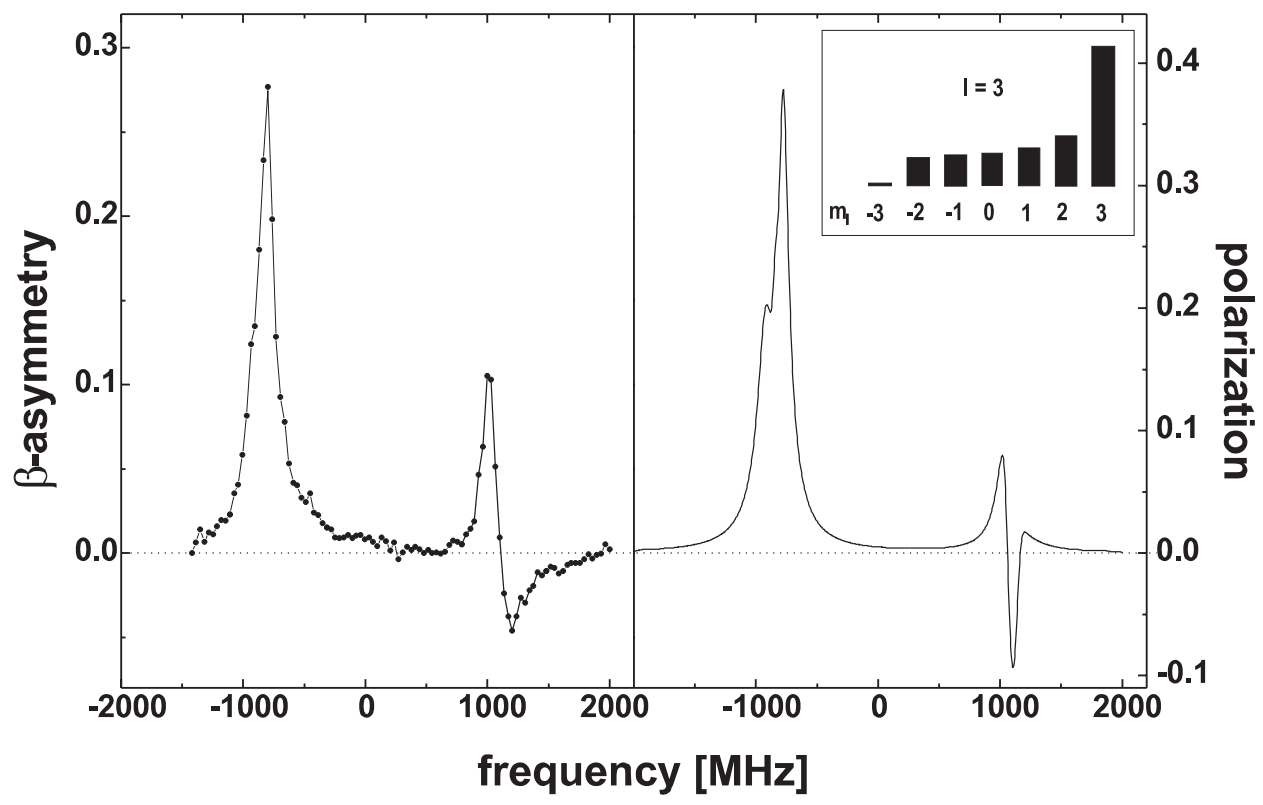

Figure 2: Hyperfine structure spectrum of ${ }^{26} \mathrm{Na}$ obtained by recording the $\beta$-decay asymmetry as a function of the acceleration voltage. Left: experimental, right: calculation according to Equation 3. The inlay shows the distribution of population of the $\mathrm{m}_{I}$ levels for the polarization maximum. 

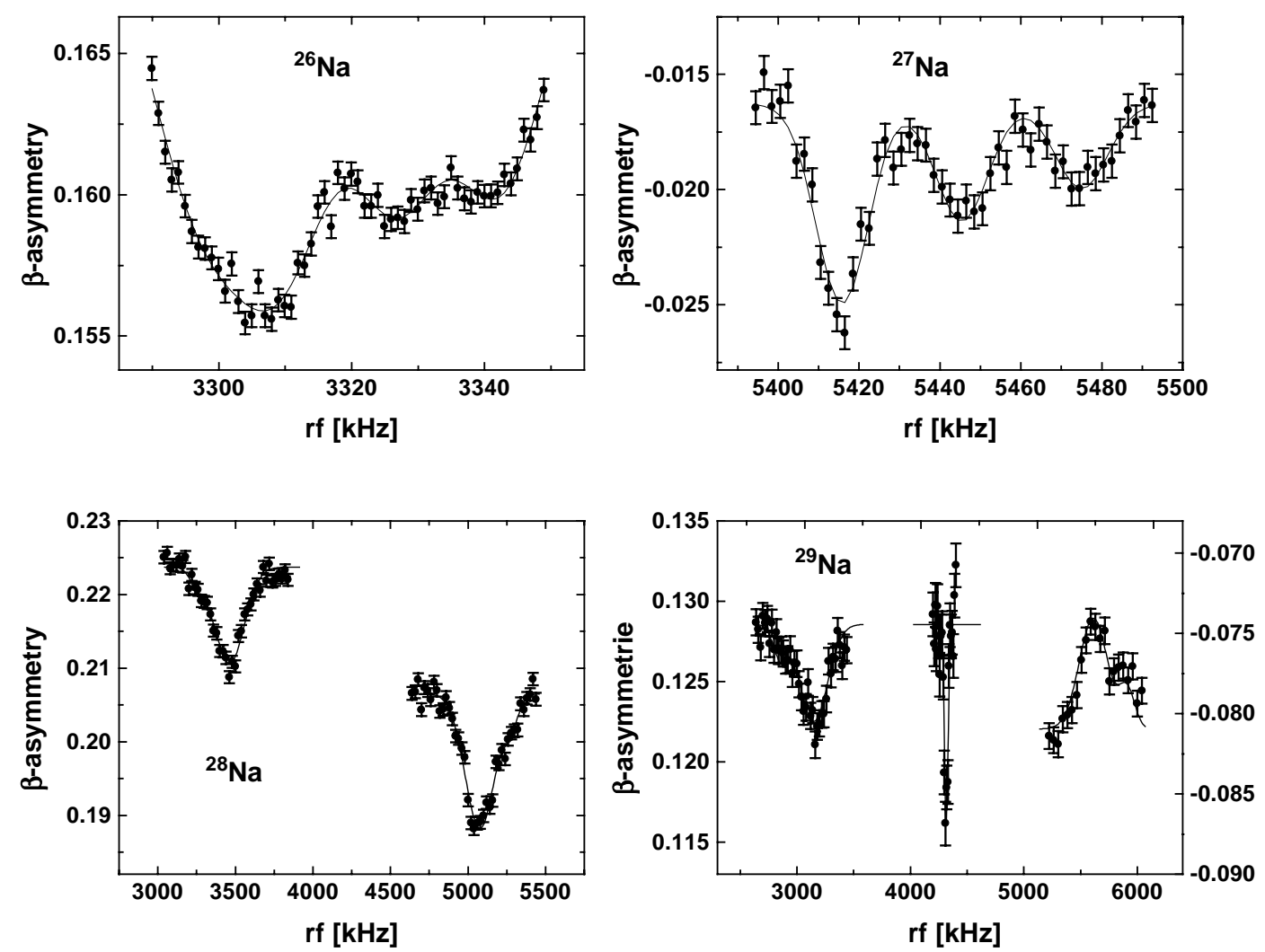

Figure 3: $\beta$-NMR spectra of ${ }^{26} \mathrm{Na},{ }^{27} \mathrm{Na},{ }^{28} \mathrm{Na}$ and ${ }^{29} \mathrm{Na}$ in $\mathrm{LiNbO}_{3}$ (for explanations see text).

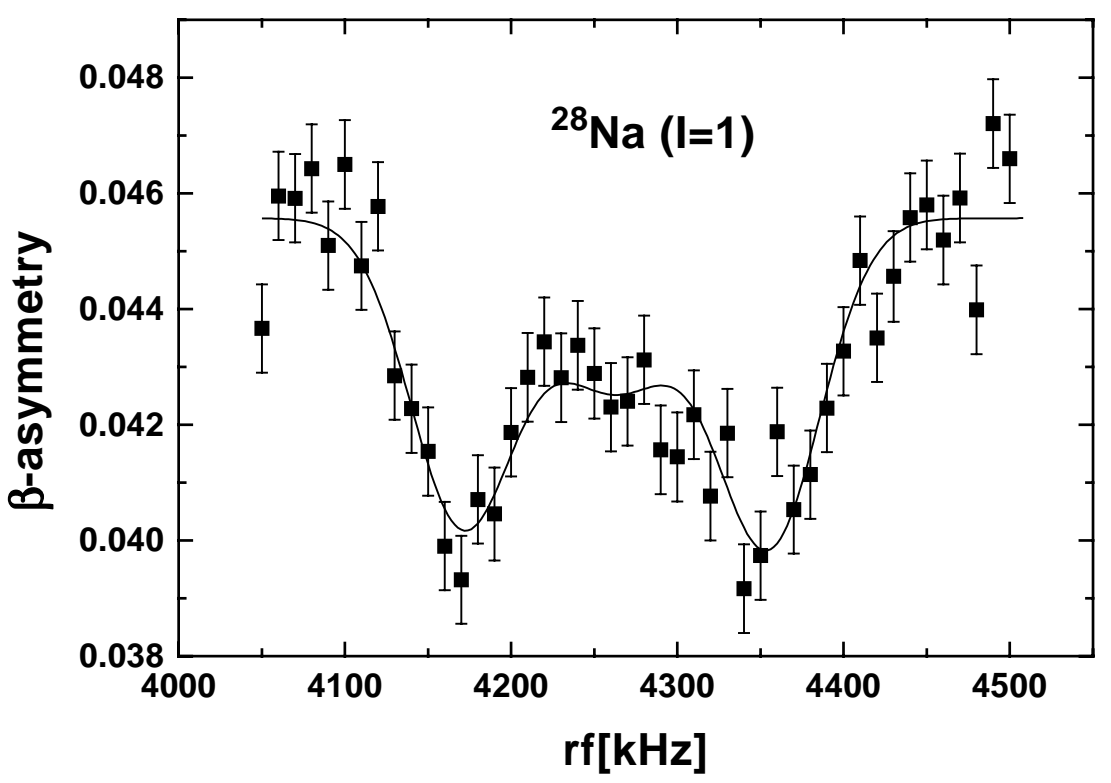

Figure 4: $\beta$-NMR spectrum of ${ }^{28} \mathrm{Na}$ in $\mathrm{NaNO}_{3}$. 


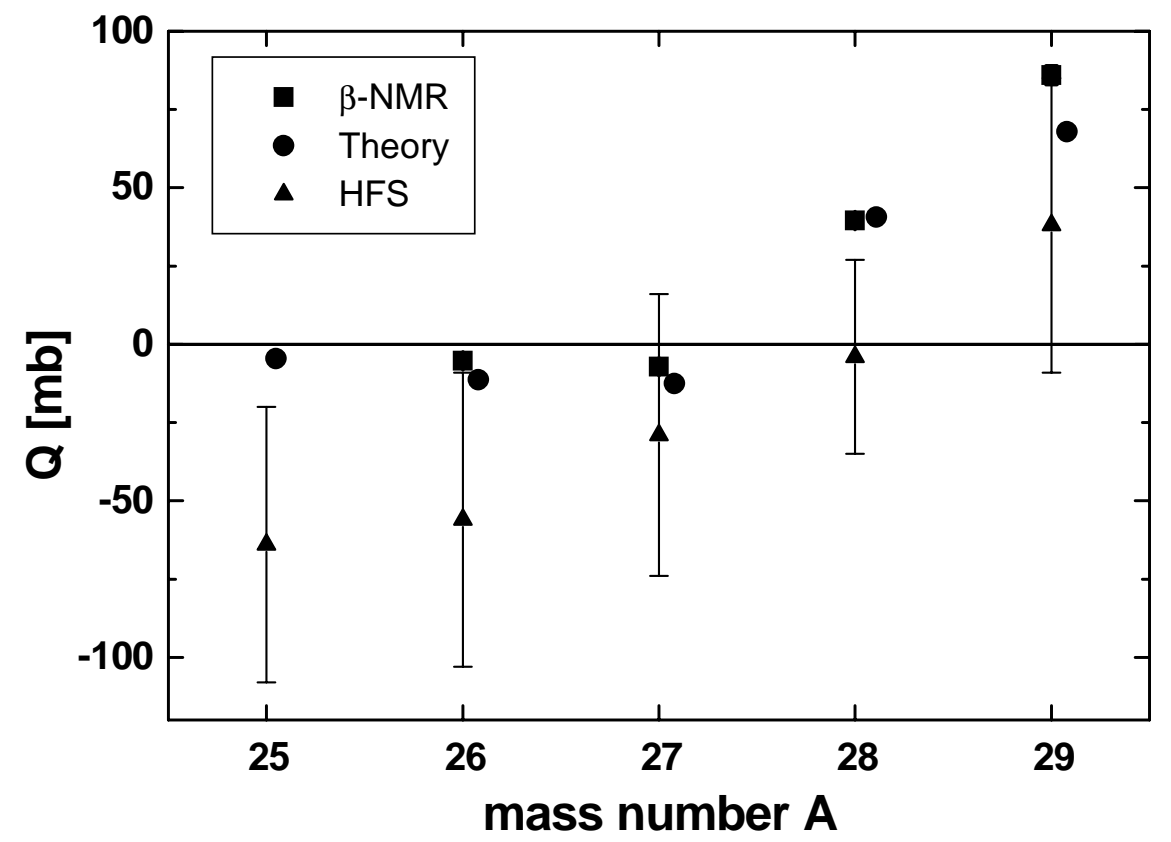

Figure 5: Experimental quadrupole moments of ${ }^{26-29} \mathrm{Na}$ compared to results reevaluated from ref. [1] and to theory. 